\title{
Do mitochondria donate membrane to form autophagosomes or undergo remodeling to form mitochondrial spheroids?
}

\author{
Wen-Xing Ding ${ }^{1 *}$ and Eeva-Liisa Eskelinen ${ }^{2}$
}

\section{To the Editor}

We read with great interest the recent paper by Cook et al. [1] in which the authors described a striking mitochondrial vesicle structure induced by some anti-cancer drugs (including Fulvestrant, an antiestrogen drug, or Imatinib, a tyrosine kinase inhibitor) in cultured cancer cells. Using electron microscopy immuno-gold labeling, they further found that some of these mitochondrial vesicles seemed to be labeled with microtubule-associated protein 1 light chain 3 (LC3), a marker for autophagosomes/autolysosomes. Therefore the authors concluded that mitochondria directly donate their membrane to form autophagosomes. We recently reported a similar mitochondrial structure change, which we named mitochondrial spheroids [2,3]. Mitochondrial spheroids are unique mitochondrial structures, which have a ring or cup-like morphology. They look similar to autophagosomes with the interior, or lumen, being surrounded by four mitochondrial membranes and squeezed mitochondrial matrix [3]. Intriguingly, we also demonstrated by confocal microscopy and immuno-gold EM analysis that mitochondrial spheroids are acidic compartments that have acquired lysosomal markers. Mitochondrial spheroids are found in cultured immortalized cells and cancer cells when they are subjected to CCCP-induced mitochondrial depolarization, a widely used mitophagy model in vitro. More importantly, we also found an increase in the number of mitochondrial spheroids in acetaminophen-treated mouse liver. Mechanistically, we found that the formation of mitochondrial spheroids requires mitochondrial fusion proteins mitofusin 1 or mitofusin 2 and is negatively regulated by Parkin, an ubiquitin E3 ligase that induces mitophagy in vitro [4]. The altered mitochondrial structures in the study by Cook et al.

\footnotetext{
* Correspondence: wxding@kumc.edu

'Department of Pharmacology, Toxicology and Therapeutics, University of Kansas Medical Center, MS 1018, 3901 Rainbow Blvd, Kansas City, KS 66160, USA

Full list of author information is available at the end of the article
}

are very similar to mitochondrial spheroids, which seems to suggest that formation of mitochondrial-formed autophagosomes/mitochondrial spheroids could be a common mitochondrial adaptation response to various mitochondrial stresses. However, there are fundamental differences in these two studies in terms of data interpretation and conclusions. One key question is whether mitochondria directly donate their membrane to form autophagosomes. The evidence for the notion that mitochondria donate their membranes to form autophagosomes was based on immuno-gold labeling using an LC3 antibody, which Cook et al. used to show that LC3 positive membranes were closely associated with mitochondrial vesicles. While these data could suggest that mitochondrial membranes were utilized to form autophagosomes, the closely-associated LC3 positive membrane could also be due to the fusion of mitochondrial vesicles with an autophagosome/autolysosome. Alternatively, it is also possible that LC3 positive membrane next to a mitochondrial vesicle could reflect the process where a mitochondron is enveloped by an autophgosome. However, it should be noted that there was no difference in the extent of formation of mitochondrial spheroids in wild type and Atg5- or Atg7-deficient cells, suggesting that the formation of mitochondrial spheroids is independent of canonical autophagy machinery [2]. Cook et al. did not test the dependency on Atg7, of the mitochondrial-formed autophagosome structures in the drug-treated cancer cells. Our second concern is the notion that mitochondrial-formed autophagosomes represent a novel mechanism of Parkin-associated mitophagy. Cook et al. found that there were very high levels of Pink1 in the cytosolic fractions in LCC9 breast cancer cells with barely detectable levels on the mitochondria after drug treatment. This is quite intriguing since it is generally thought that Pink1 is a mitochondrial serine/threonine protein kinase [5-7]. Moreover, it is well known that Parkin has a tumor suppressor function and is generally silenced and its expression is lost in many immortalized and transformed 
cancer cells such as HeLa cells [8-10]. Many of the Parkinmediated mitophagy studies in cultured cells rely on the ectopical overexpression of Parkin [9,11]. Therefore, it is very interesting that Cook et al. found that LCC9 breast cancer cells had endogenous Parkin expression although it was unclear how these LCC9 cancer cells were different from other immortalized/cancerous cells. Moreover, it was also not clear how many of the mitochondrial-formed autophagosomes were labeled Parkin positive since no quantitative data were provided. Another unresolved issue for both mitochondrial spheroids and mitochondrialformed autophagosomes are their unclear physiological roles. While we have proposed that the formation of mitochondrial spheroids may represent an alternative mechanism for regulating mitochondrial homeostasis and thus to limit drug-induced cell death, more future studies are definitely needed to further clarify this notion by using genetic mitofusin 1 or mitofusin 2 knockout cells in which mitochondrial spheroids cannot form. In the study by Cook at al, it was not clear whether the formation of the mitochondrial-formed autophagosomes served as mitochondrial quality control to protect the cells from anticancer drug-induced cell death since this aspect was not addressed. Taken together, it seems that more studies are needed to further confirm whether mitochondria donate their membrane to form autophagosomes as proposed in the Cook et al. study, or whether mitochondria are turned over by forming spheroids as proposed by our study $[2,3]$, or whether both alternatives are possible depending on the context. However, it can be concluded that certain drugs that may damage mitochondria can induce dramatic mitochondrial structural remodeling to form vesicular structures/mitochondrial spheroids. However, the physiological or pathological significance of these unique mitochondrial structures remains to be further studied.

\section{Author details}

'Department of Pharmacology, Toxicology and Therapeutics, University of Kansas Medical Center, MS 1018, 3901 Rainbow Blvd, Kansas City, KS 66160, USA. ${ }^{2}$ Department of Biosciences, Division of Biochemistry and

Biotechnology, The University of Helsinki, 00014 Helsinki, Finland.

Received: 4 September 2014 Accepted: 20 October 2014

Published: 14 November 2014

\section{References}

1. Cook KL, Soto-Pantoja DR, Abu-Asab M, Clarke PA, Roberts DD, Clarke R: Mitochondria directly donate their membrane to form autophagosomes during a novel mechanism of parkin-associated mitophagy. Cell Biosci 2014, 4:16

2. Ding WX, Guo F, Ni HM, Bockus A, Manley S, Stolz DB, Eskelinen EL, Jaeschke $H$, Yin XM: Parkin and mitofusins reciprocally regulate mitophagy and mitochondrial spheroid formation. J Biol Chem 2012 287:42379-42388.

3. Ding WX, Li M, Biazik JM, Morgan DG, Guo F, Ni HM, Goheen M, Eskelinen EL, Yin XM: Electron microscopic analysis of a spherical mitochondrial structure. J Biol Chem 2012, 287:42373-42378.

4. Yin $X M$, Ding $W X$ : The reciprocal roles of PARK2 and mitofusins in mitophagy and mitochondrial spheroid formation. Autophagy 2013, 9:1687-1692.
5. Yamano K, Youle RJ: PINK1 is degraded through the N-end rule pathway. Autophagy 2013, 9:1758-1769.

6. Lazarou M, Jin SM, Kane LA, Youle RJ: Role of PINK1 binding to the TOM complex and alternate intracellular membranes in recruitment and activation of the E3 ligase Parkin. Dev Cell 2012, 22:320-333.

7. Jin SM, Youle RJ: PINK1- and Parkin-mediated mitophagy at a glance. J Cell Sci 2012, 125:795-799.

8. $\mathrm{Xu} \mathrm{L}$, Lin DC, Yin D, Koeffler HP: An emerging role of PARK2 in cancer. J Mol Med (Berl) 2014, 92:31-42.

9. Ding WX, Ni HM, Li M, Liao Y, Chen X, Stolz DB, Dorn GW 2nd, Yin XM: Nix is critical to two distinct phases of mitophagy, reactive oxygen speciesmediated autophagy induction and Parkin-ubiquitin-p62-mediated mitochondrial priming. J Biol Chem 2010, 285:27879-27890.

10. Gong Y, Zack TI, Morris LG, Lin K, Hukkelhoven E, Raheja R, Tan IL, Turcan S, Veeriah S, Meng S, Viale A, Schumacher SE, Palmedo P, Beroukhim R, Chan TA: Pan-cancer genetic analysis identifies PARK2 as a master regulator of G1/S cyclins. Nat Genet 2014, 46:588-594.

11. Narendra D, Tanaka A, Suen DF, Youle RJ: Parkin is recruited selectively to impaired mitochondria and promotes their autophagy. J Cell Biol 2008, 183:795-803.

doi:10.1186/2045-3701-4-65

Cite this article as: Ding and Eskelinen: Do mitochondria donate membrane to form autophagosomes or undergo remodeling to form mitochondrial spheroids? Cell \& Bioscience 2014 4:65.

\section{Submit your next manuscript to BioMed Central and take full advantage of:}

- Convenient online submission

- Thorough peer review

- No space constraints or color figure charges

- Immediate publication on acceptance

- Inclusion in PubMed, CAS, Scopus and Google Scholar

- Research which is freely available for redistribution 